\title{
ON-LINE CHANGE MONITORING WITH TRANSFORMED MULTI-SPECTRAL TIME SERIES, A STUDY CASE IN TROPICAL FOREST
}

\author{
Meng $\mathrm{Lu}^{\mathrm{a}, *}$, Eliakim Hamunyela ${ }^{\mathrm{b}}$ \\ ${ }^{\text {a } I n s t i t u t e ~ f o r ~ G e o i n f o r m a t i c s, ~ W e s t f a ̈ l i s c h e ~ W i l h e l m s-U n i v e r s i t t ~ M u ̈ n s t e r ~(W W U), ~ H e i s e n b e r g s t r a ß e ~ 2, ~} 48149$ Münster, Germany \\ - meng.lu@uni-muenster.de \\ ${ }^{\mathrm{b}}$ Laboratory of Geo-Information Science and Remote Sensing, Wageningen University, Droevendaalsesteeg 3, Wageningen 6708 PB, \\ The Netherlands \\ - eliakim.hamunyela@wur.nl
}

KEY WORDS: Multi-Spectral, BFAST, Dimension Reduction, Deforestation Monitor

\begin{abstract}
:
In recent years, the methods for detecting structural changes in time series have been adapted for forest disturbance monitoring using satellite data. The BFAST (Breaks For Additive Season and Trend) Monitor framework, which detects forest cover disturbances from satellite image time series based on empirical fluctuation tests, is particularly used for near real-time deforestation monitoring, and it has been shown to be robust in detecting forest disturbances. Typically, a vegetation index that is transformed from spectral bands into feature space (e.g. normalised difference vegetation index (NDVI)) is used as input for BFAST Monitor. However, using a vegetation index for deforestation monitoring is a major limitation because it is difficult to separate deforestation from multiple seasonality effects, noise, and other forest disturbance. In this study, we address such limitation by exploiting the multi-spectral band of satellite data. To demonstrate our approach, we carried out a case study in a deciduous tropical forest in Bolivia, South America. We reduce the dimensionality from spectral bands, space and time with projective methods particularly the Principal Component Analysis (PCA), resulting in a new index that is more suitable for change monitoring. Our results show significantly improved temporal delay in deforestation detection. With our approach, we achieved a median temporal lag of 6 observations, which was significantly shorter than the temporal lags from conventional approaches (14 to 21 observations).
\end{abstract}

\section{INTRODUCTION}

Near real-time change monitoring has important application in forest management. Open access to satellite data (e.g Landsat and Sentinels) enables for near real-time detection of forest disturbance using advanced time series analysis methods (Banskota et al., 2014; Forkel et al., 2013; Kuan and Hornik, 1995). Most of the recent studies on near real-time forest disturbance monitoring have applied BFAST Monitor (Verbesselt et al., 2012), which detects forest disturbances by identifying a historical period, fit a linear regression model for historical time series, and monitor change in newly acquired observation either with a cumulative sum (CUSUM) or moving sums (MOSUM) process of the differences between new data and model predictions. Studies applying BFAST monitor mostly use normalised difference vegetation index (NDVI), normalised difference moisture index (NDMI), and enhanced vegetation index (EVI). These indices contrast the absorption and reflection properties of vegetation between near or short-wave infrared bands and visible bands.

Monitoring deforestation on single vegetation index has several limitations: 1) the harmonic model might not be sufficient to capture the complex seasonality in tropical forest, 2) one model is not sufficient for spatially heterogeneous tree species. 3) deforestation can be hard to separate from drought, other disturbances, and noise, 4) training data is required to identify if the detected change is deforestation. Recent studies attempted to solve the limitation (3) by integrating data from two sensors and introducing a climate variable (Dutrieux et al., 2015), or attempted to reduce the effect of multiple seasonality with a vegetation index that is normalized to spatially neighboring pixels (Hamunyela et al., 2016).
In this study we integrate the spectral information in BFAST Monitor change monitoring framework and show the potential of integrating multidimensional information to improve the change monitoring process. The exploration is based on the assumption that the original variables can be seen as the composite of independent variables, with one of them containing deforestation information.

\section{STUDY AREA AND DATA PREPROCESSING}

We carried out a case study in a deciduous tropical forest in Bolivia, South America $\left(18.49^{\circ} \mathrm{S}, 62.36^{\circ} \mathrm{W}, 10,000 \mathrm{~km}^{2}\right)$. The dataset consists of all available Landsat TM5 and Landsat ETM +7 L1T imagery before 2015. All the sample points were collected manually with high and medium spatial resolution satellite data. We masked the snow, shadow and water and cloud with FMask (Zhu and Woodcock, 2012). Low extreme values are filtered and interpolated, and Band values that are out of the range (1-10000) are filtered.

\section{METHOD}

\subsection{Multi-spectral bands transformation}

We use Principal Component Analysis (PCA) to find a projecting vector $a$ on the original variables matrix $X$, so that the new variables in $a X$ are orthogonal. This means to diagonalize the co-variance matrix of $X(\operatorname{var}(X))$,

\footnotetext{
${ }^{*}$ Corresponding author
}

$\operatorname{var}(a X)=a^{\prime} \Sigma a$ 


$$
\Sigma a=\lambda a,
$$

where $a$ is the projecting matrix, and $\lambda$ is the diagonalized covariance matrix that is sorted from the largest value of the variance. This lead to the eigen-vector based analysis, where $a$ contains eigenvector or the PC loadings, and $\lambda$ contains eigenvalue. The new variable matrix is then obtained as the weighted combination of original variables, which is the PC score.

PC loadings are trained on matrix $X$ contains the spectral time series of Landsat image band $1-5$, and band 7 for spectral time series of each location, $X=\left[X_{i j}\right]_{(m \times 6)}$, with the gray scale value at time $i(i=1, \ldots, m)$, for each spectral band $j(j=1, \ldots, 6) . X$ is centered to zero mean and standardized to unit variance.

The input matrix $X$ is all available data, with this method we see the original spectral bands as mixing variables, and expect to find the independent data source that compose to this variable:

$$
X=A S,
$$

where $\mathrm{X}$ denotes the original data, A denotes a mixing matrix, and $\mathrm{S}$ denotes the independent components.

The goal then is to estimate a separation matrix to separate the independent sources from the mixture.

$$
Y=W X
$$

where $\mathrm{Y}$ denotes the independent data source, $\mathrm{W}$ denotes the separation matrix, and $\mathrm{X}$ denotes the original data.

\subsection{Deforestation Monitoring}

We applied BFAST monitor on the scores of PC components to monitor deforestation. To model the temporal behaviour of the forest, we only fit a mean model to the time series because the seasonality effects have been removed. Data covering the period of 1984 - 2005 were used as initial stable history. Then the algorithms sequentially monitor for change in the monitoring period. If the monitoring period becomes longer than 2 years, and no change is detected, the data that have been monitored are then moved into stable historical period. The algorithms stops monitoring when change is detected or the test reach to the last observation. The results were validated considering spatial accuracy and temporal accuracy. The spatial accuracy consists of omission error, commission error, and overall accuracy. The temporal accuracy is measured by the number of observations between BFAST detected change date and real change date. This is to cope with the irregularity of Landsat data. We compared our results to the results achieved from NDMI. NDMI has been recommended in recent studies because it achieve best results. NDMI is calculated as (SWIF-NIR)/(SWIF+NIR). We fitted a 1st order harmonic model om NDMI time series when monitoring deforestation using BFAST Monitor.

\section{RESULT}

Our results show that using the score of PC 3 has resulted in significantly improved overall accuracy and a shorter temporal delay than when monitoring deforestation using NDMI index(Table 1). Time series of different indices and the results of disturbance detection can be observed from (Figure 1. Using NDMI has resulted in many false positives (change detected before real change). The score of PC3 successfully extracted the signal of the disturbance, and thus resulted in a more suitable index for deforestation monitoring.

Table 1: validation result

\begin{tabular}{ccc}
\hline \hline & overall spatial accuracy & temporal delay \\
\hline PC1 & 0.813 & 13 \\
PC2 & 0.692 & 22 \\
PC3 & 0.900 & 7 \\
NDMI & 0.331 & 22 \\
\hline
\end{tabular}

Table 2: confusion matrix. Temporal detection delay is by counting the number of observation. PCA3 is the score of the 3rd PC that is applied on all the available data. PCA3 history is to compute PCA on history period only.

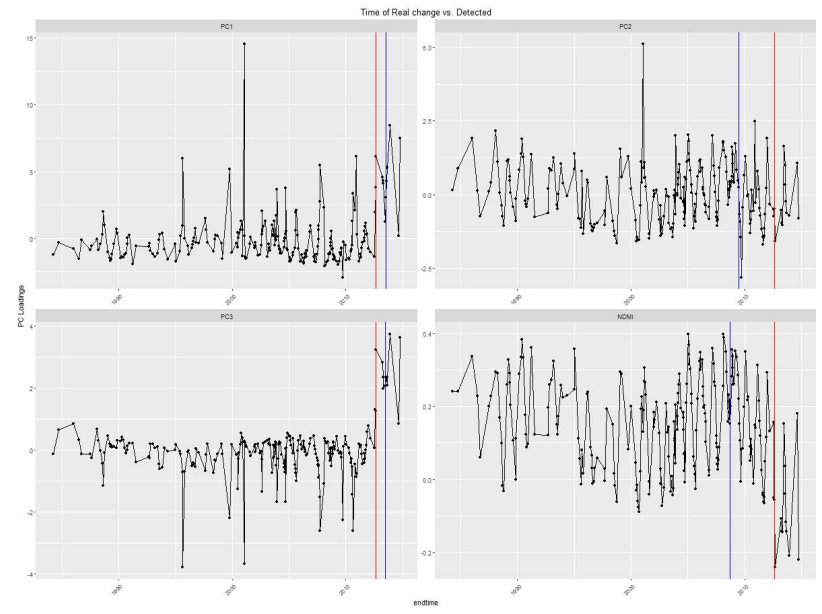

Figure 1: time of real change (red line) vs. time of detected change (blue line) using the PCA scores as index and NDMI as index

\section{DISCUSSION}

\subsection{The PCA integrated BFAST}

In this study we still used the BFAST framework for deforestation monitor. The methodology is different from the original idea of monitoring an vegetation index that fluctuate with the change of vegetation, which is subject to drought and seasonality effects. The proposed method is to find the source variables of mixing variable, which the source variables can be estimated with different ways. Such as a variety of models in factor analysis. In the situation where the distribution of original variables variables are not Gaussian, and the non-Gaussian change is of interest, ICA (independent component analysis) might be used. This PCA integrated method detects the change of relationship between multispectral bands, which is free from the modeling or removing of seasonality, and other short-term climatic effects.

\section{2 limitation}

The limitation of this method is that the PC components are ordered by eigenvalues (correlation), which does not contain physical meanings. For example, the pattern of the eigenvectors of the 2nd PC component of one time series could show the same pattern to the eigenvectors of the 1st PC component of another time 
series. The method is successful here because forest is spatially homogeneous (consists only vegetation), so that for most of the time series the order of PC components would be consistent.

\section{CONCLUSION}

In this paper we firstly explored the transformation of spectral time series into new variables that include a clearer change signal. We distinguish this method between the previously feature space transformation methods (e.g. NDMI), which does not use the previous time series information. Secondly we explored a way to remove seasonality effect. Both results indicate improved deforestation monitor in temporal delay. With increased spatial and temporal resolution of satellite data in future, combining dimensional reduction methods and sequential test of time series structural change has promising application in realizing near realtime deforestation monitor and contribute greatly to forest management and ecosystem conservation.

\section{References}

Banskota, A., Kayastha, N., Falkowski, M. J., Wulder, M. A., Froese, R. E. and White, J. C., 2014. Forest monitoring using landsat time series data: A review. Canadian Journal of Remote Sensing 40(5), pp. 362-384.

Dutrieux, L. P., Verbesselt, J., Kooistra, L. and Herold, M., 2015. Monitoring forest cover loss using multiple data streams, a case study of a tropical dry forest in bolivia. ISPRS Journal of Photogrammetry and Remote Sensing 107, pp. 112-125.

Forkel, M., Carvalhais, N., Verbesselt, J., Mahecha, M. D., Neigh, C. S. and Reichstein, M., 2013. Trend change detection in NDVI time series: Effects of inter-annual variability and methodology. Remote Sensing 5(5), pp. 2113-2144.

Hamunyela, E., Verbesselt, J. and Herold, M., 2016. Using spatial context to improve early detection of deforestation from landsat time series. Remote Sensing of Environment 172, pp. 126138.

Kuan, C.-M. and Hornik, K., 1995. The generalized fluctuation test: A unifying view. Econometric Reviews 14(2), pp. 135161.

Verbesselt, J., Zeileis, A. and Herold, M., 2012. Near real-time disturbance detection using satellite image time series. Remote Sensing of Environment 123, pp. 98-108.

Zhu, Z. and Woodcock, C. E., 2012. Object-based cloud and cloud shadow detection in landsat imagery. Remote Sensing of Environment 118, pp. $83-94$. 